\title{
Mass Spectrometric Identification of Human Prostate Cancer-derived Proteins in Serum of Xenograft-bearing Mice*
}

\author{
Gert-Jan C. M. van den Bemd‡, Jeroen Krijgsveld§, Theo M. Luiderף, \\ Angelique L. van Rijswijkł, Jeroen A. A. Demmers\|, and Guido Jenster ${ }^{\star \star}$
}

Lack of sensitivity and specificity of current tumor markers has intensified research efforts to find new biomarkers. The identification of potential tumor markers in human body fluids is hampered by large variability and complexity of both control and patient samples, laborious biochemical analyses, and the fact that the identified proteins are unlikely produced by the diseased cells but are due to secondary body defense mechanisms. In a new approach presented here, we eliminate these problems by performing proteomic analysis in a prostate cancer xenograft model in which human prostate cancer cells form a tumor in an immune-incompetent nude mouse. Using this concept, proteins present in mouse serum that can be identified as human will, by definition, originate from the human prostate cancer xenograft and might have potential diagnostic and prognostic value. Using one-dimensional gel electrophoresis, liquid chromatography, and mass spectrometry, we identified tumor-derived human nm23/nucleoside-diphosphate kinase (NME) in the serum of a nude mouse bearing the androgen-independent human prostate cancer xenograft PC339. NME is known to be involved in the metastatic potential of several tumor cells, including prostate cancer cells. Furthermore we identified six human enzymes involved in glycolysis (fructose-bisphosphate aldolase A, triose-phosphate isomerase, glyceraldehyde-3-phosphate dehydrogenase, $\alpha$ enolase, and lactate dehydrogenases A and B) in the serum of the tumor-bearing mice. The presence of human NME and glyceraldehyde-3-phosphate dehydrogenase in the serum of PC339-bearing mice was confirmed by Western blotting. Although the putative usefulness of these proteins in predicting prognosis of prostate cancer remains to be determined, the present data illustrate that our approach is a promising tool for the focused discovery of new prostate cancer biomarkers. Molecular \& Cellular Proteomics 5:1830-1839, 2006.

From the $\ddagger$ Department of Urology, Josephine Nefkens Institute and Departments of ףNeurology and ||Biochemistry, Center for Biomics, Erasmus MC, 3000 DR, Rotterdam and §Department of Biomolecular Mass Spectrometry, Bijvoet Center for Biomolecular Research and Utrecht Institute for Pharmaceutical Sciences, Utrecht University, 3584 CA, Utrecht, The Netherlands

Received, November 14, 2005, and in revised form, March 10, 2006

Published, MCP Papers in Press, May 19, 2006, DOI 10.1074/ mcp.M500371-MCP200
Most clinically established tumor markers (e.g. prostatespecific antigen (PSA), ${ }^{1}$ cancer antigen CA125, $\alpha$-fetoprotein, human chorionic gonadotropin, carcinoembryonic antigen, and cytokeratins) are proteins produced by cancer cells. Indeed cancer cell-derived proteins are considered the best markers to determine the presence, size, status, and progression of the tumor. Still there is an urgent need for new markers with high sensitivity and specificity that can detect disease in its earliest stage and that predict disease progression. Currently for prostate cancer, blood levels of PSA are the key marker. Dependent on age, levels greater than $2-4 \mathrm{ng} / \mathrm{ml}$ are considered to be elevated but are limited, however, in distinguishing prostate cancer from benign prostatic hyperplasia or prostate inflammation $(1,2)$. In addition, $\sim 25 \%$ of men diagnosed with prostate cancer have a PSA level below $4 \mathrm{ng} / \mathrm{ml}$ (1, 3). More important than prostate cancer diagnosis alone is to determine whether a diagnosed prostate tumor will become life-threatening or will remain clinically insignificant. Many older men will have insignificant in situ prostate tumors causing positive diagnostic results. Treatment of these cancers causes more harm than it saves lives. Overtreatment is a serious problem, and new prognostic biomarkers that are better in determining which cancers will be severe and merit treatment are indispensable (4).

Most biomarker discovery protocols currently utilized compare series of serum or other body fluid from subjects with and without disease using SELDI/MALDI mass spectrometry. These explorations for potential markers in human body fluids are hampered by (i) large sample variation and thus the need for large numbers of patient samples, (ii) the high complexity and wide dynamic range of proteins in biological samples and, as a consequence, the need for laborious biochemical analyses to identify the protein marker, and (iii) the fact that the identified proteins are unlikely produced by the diseased cells but are due to secondary body defense mechanisms. We have developed and tested a novel method to instantly detect and identify proteins in body fluids that are produced by the diseased cells. In the discovery procedure we utilize a xenograft model system in which human prostate cancer cells

${ }^{1}$ The abbreviations used are: PSA, prostate-specific antigen; ALDA, fructose-bisphosphate aldolase A; GAPD, glyceraldehyde-3phosphate dehydrogenase; LDH, lactate dehydrogenase; NME, nm23/nucleoside-diphosphate kinase; PC339, prostate cancer xenograft 339; PGAM1, phosphoglycerate mutase 1; TPI, triose-phosphate isomerase. 
form a tumor in an immune-incompetent nude mouse (5). These tumor xenotransplantation models have clearly demonstrated their value for studying tumor growth and progression (6). In the grafted animal, (potential) tumor markers derived from the human cancer cells can be identified and monitored without the large variation observed in a patientbased approach.

Only a limited number of studies have focused on the potential power of xenograft models to study the serum expression of tumor-associated proteins in a proteome-broad approach. In the serum of mice xenotransplanted with human mammary carcinoma cell lines, Roemer et al. (7) identified mouse $\beta$-haptoglobin. Although $\beta$-haptoglobin was also detected in the mock-transplanted animals, the expression in the tumor-bearing mice was sustained for a longer period of time and showed differential terminal glycan modifications in the tumor-bearing mice. Obviously this acute phase protein was not originating from the tumor, but its altered expression was a direct consequence from the host response to the tumor. Overexpression of mouse haptoglobin and other acute phase proteins was also observed in another proteomic plasma screening of mice inoculated with human cancer cell lines derived from stomach, nasopharyngeal, colon, oral, or brain cancers (8). Recently a study by Hood et al. (9) demonstrated a powerful method to identify potential biomarkers in a lung cancer xenograft mouse model. Although numerous proteins were identified, also in this study no identification of a human-specific, tumor-derived protein was reported.

In the present study, we used a proteomic approach to investigate the presence of human proteins in the serum of a mouse carrying an androgen-independent prostate cancer xenograft (PC339). One of the crucial aspects to make our study successful is the ability to distinguish mouse from human proteins in a complex mixture. This is no trivial task given the high homology of many proteins from both species. However, using a mass spectrometer with high accuracy and sensitivity, we demonstrated proof of concept by the identification of several human proteins: nm23/nucleoside-diphosphate kinase (NME), known to be involved in the regulation of cancer cell progression and metastasis $(10,11)$, and the glycolytic enzymes fructose-bisphosphate aldolase A (ALDA), triose-phosphate isomerase (TPI), liver and muscle glyceraldehyde-3-phosphate dehydrogenase (GAPD), $\alpha$ enolase, and lactate dehydrogenases $A$ and $B$ (LDH-A and $-B$ ).

\section{EXPERIMENTAL PROCEDURES}

A general scheme of the procedure is depicted in Fig. 1.

Animals-For our studies we used the human PC339 prostate cancer xenograft model system described by Van Weerden et al. (5). Immune-incompetent athymic male nude (nu/nu) BALB/c mice were obtained from Taconic, Ry, Denmark. To obtain control mouse serum, eight drops of blood were collected by retro-orbital puncture. Then these same mice were inoculated subcutaneously at both shoulders with androgen-independent PC339 human prostate cancer cells. After 4-5 weeks, blood was collected from the tumor-bearing mice by retro-orbital puncture, and the mice were sacrificed. For the control and xenograft-bearing groups, separate pools from nine individual serum samples were prepared and stored at $-80^{\circ} \mathrm{C}$. The experimental protocol was approved by the Animal Experiments Committee under the national Experiments on Animals Act and adhered to the rules laid down in this national law that serves the implementation of "Guidelines on the protection of experimental animals" by the Council of Europe (1986), Directive 86/609/EC.

Removal of High Abundance Proteins-After dilution and filtration, the three most abundant serum proteins (albumin, immunoglobulins, and transferrin) were removed from the mouse serum utilizing multiple affinity removal spin cartridges (Agilent Technologies, Wilmington, $\mathrm{DE})$ according to the manufacturer's instructions. Following depletion, samples were concentrated on 5-kDa-cutoff ultracentrifugation columns (Vivascience, Hannover, Germany). Total protein concentration was determined by the Bradford method with commercially available Bradford reagents (Bio-Rad) using bovine serum albumin as a standard.

One-dimensional Gel Electrophoresis - The depleted serum samples (10 $\mu \mathrm{g}$ of protein) were mixed with Laemmli sample buffer (1:1) and separated by SDS-PAGE (Mini-Protean III, Bio-Rad) on precast 4-20\% polyacrylamide linear gradient gels (Bio-Rad) using Tris-glycine buffer, $\mathrm{pH}$ 8.6, as the running buffer. Prestained high range molecular weight markers (SeeBlue, Invitrogen) were loaded on each gel. Gels were either blotted onto Protran nitrocellulose membranes (Schleicher \& Schuell) or stained using Coomassie Brilliant Blue (Merck).

In-gel Alkylation and Digestion-Lanes were excised, and the gel encompassing a range of $5-50 \mathrm{kDa}$ was cut into $1-\mathrm{mm}$ segments using a long blade gel slicer (Mickle Laboratory Engineering Co., Gomshall, Surrey, UK). Three gel slices were pooled, washed, and destained twice with $50 \%(\mathrm{v} / \mathrm{v})$ acetonitrile in $50 \mathrm{~mm}$ ammonium bicarbonate overnight at $4{ }^{\circ} \mathrm{C}$. After dehydration in $100 \%$ acetonitrile, samples were reduced with $6.5 \mathrm{~mm}$ DTT in $50 \mathrm{~mm}$ ammonium bicarbonate for $1 \mathrm{~h}$ at $37^{\circ} \mathrm{C}$. After alkylation with $54 \mathrm{~mm}$ iodoacetamide in $50 \mathrm{~mm}$ ammonium bicarbonate, proteins were dehydrated in $100 \%$ acetonitrile and then rehydrated with the digestion solution containing $10 \mathrm{ng} / \mu \mathrm{l}$ ultragrade sequencing trypsin (Promega, Madison, WI) for 30 $\mathrm{min}$ at room temperature. After addition of $30 \mu \mathrm{l}$ of $50 \mathrm{~mm}$ ammonium bicarbonate solution, gel particles were incubated overnight at $37^{\circ} \mathrm{C}$. The peptides were extracted using $0.5 \%$ formic acid in $50 \%$ acetonitrile, dried completely in a vacuum centrifuge, and stored at $-80^{\circ} \mathrm{C}$ until analysis.

Liquid Chromatography-Mass Spectrometry and Database Searching-Nano-LC-MS/MS analyses were performed on a nanoflow LC system (CapLC, Waters) coupled to a Q-TOF Ultima mass spectrometer (Waters). Peptide mixtures were trapped on a Jupiter ${ }^{\mathrm{TM}} \mathrm{C}_{18}$ reversed phase column (Phenomenex; column dimensions, $1.5 \mathrm{~cm} \times$ $100 \mu \mathrm{m}$, packed in-house) at a flow rate of $7 \mu \mathrm{l} / \mathrm{min}$. Peptides were separated using a Jupiter $\mathrm{C}_{18}$ reversed phase column (Phenomenex; column dimensions, $15 \mathrm{~cm} \times 50 \mu \mathrm{m}$, packed in-house) and a gradient of $0-80 \%$ acetonitrile in $0.1 \mathrm{~m}$ acetic acid in $70 \mathrm{~min}$ and at a constant flow rate of $200 \mathrm{nl} / \mathrm{min}$. Fragmentation of the peptides was performed in data-dependent mode, and mass spectra were acquired in continuum mode.

For further identification, these samples were analyzed by FT-ICRMS. Nanoflow LC tandem mass spectrometry was performed by coupling an Agilent 1100 HPLC system (Agilent Technologies), operated as described before (12), to a 7-tesla LTQ-FT ${ }^{\mathrm{TM}}$ mass spectrometer (Thermo Electron, Bremen, Germany). The mass spectrometer was operated in data-dependent mode, automatically switching between MS and MS/MS acquisition. Full scan MS spectra were acquired by FT-ICR with a resolution of 20,000 at a target value of $2,000,000$. The three most intense ions were then isolated for accurate mass measurements by a FT-ICR selected ion monitoring scan that consisted of $10-\mathrm{Da}$ mass range with a resolution of 50,000 at a 
target accumulation value of 50,000 . These ions were then fragmented in the linear ion trap using collision-induced dissociation at a target value of 15,000 . MS/MS data from each LC run were converted to a single file in Mascot generic format.

For protein identification, database searches were performed using Mascot Version 2.0 (Matrix Science, London, UK) allowing 100-ppm (Q-TOF) or 5-ppm (FT-ICR-MS/MS) mass deviation for the precursor ion. A maximum number of one missed cleavage was allowed, and carbamidomethylated cysteine and oxidized methionine were set as optional modifications. Individual Mascot scores for each peptide MS/MS spectrum were $>30$. Before we annotated a certain peptide as human, a stringent selection procedure was followed. Peptide mass values were searched against the Swiss-Prot database. The peptides annotated as human in the Swiss-Prot database were re-searched against the International Protein Index (IPI) mouse database. Peptides also found in the mouse IPI database are obviously not considered as uniquely human, i.e. are not necessarily tumor-derived. Peptides that were not identified in the mouse IPI database had to be confirmed as human in the human IPI database. Additionally to check organism specificity, the human- or mouse-specific peptides were blasted against the National Center for Biotechnology Information (NCBI) database.

Western Blot Analysis-The proteins present in $7 \mu$ l of depleted mouse serum were electrophoretically separated by SDS-PAGE and blotted onto Protran nitrocellulose membrane as described above. Purified recombinant human NME1 and NME2 were used as standard. Recombinant NME1 and NME2 as well as rabbit anti-human antibodies against these proteins were a gift from Dr. Lascu, University of Bordeaux. Goat anti-rabbit horseradish peroxidase-conjugated antibody (DakoCytomation, Glostrup, Denmark) and a chemiluminescence detection kit (Roche Applied Science) were used to visualize NME1 and NME2. GAPD was detected using a mouse monoclonal anti-human GAPD antibody (LabFrontier, Seoul, South Korea) and a polyclonal goat anti-mouse horseradish peroxidase-conjugated antibody (DakoCytomation). Total cell extract of human prostate cancer PC3 cells (obtained from the American Type Culture Collection, Manassas, VA) served as a positive control, and normal mouse liver extract served as a negative control for human GAPD expression. Bands were quantified using ImageQuant Version 5.0 software (GE Healthcare).

\section{RESULTS}

Serum Protein Expression in Control and Xenograft-bearing Mice-Using the procedure schematically depicted in Fig. 1, serum protein expression of control mice and mice carrying a PC339 human prostate cancer xenograft was investigated. All nine animals developed tumors at both shoulders. Total tumor weight varied between 1,090 and 3,640 $\mathrm{mg}$ (average \pm S.E., $1,847 \pm 292 \mathrm{mg})$.

A pooled serum sample of each group was separated on a one-dimensional SDS-PAGE gel. We focused on the 5-60$\mathrm{kDa}$ region of this gel and identified over 100 different proteins by Q-TOF-MS/MS. We analyzed the same samples by FTICR-MS/MS and identified all the proteins found in the QTOF-MS/MS analysis. In addition, FT-ICR-MS/MS analysis revealed over 300 additional proteins. Although most of these proteins $(51 \%)$ were observed in both control and PC339 samples, a considerable number of proteins were only found in either the control $(18 \%)$ or the xenograft $(31 \%)$ serum sample. For instance, 13 members of the crystallin family were only observed in the control serum and not in the serum of the xenograft mice.
A

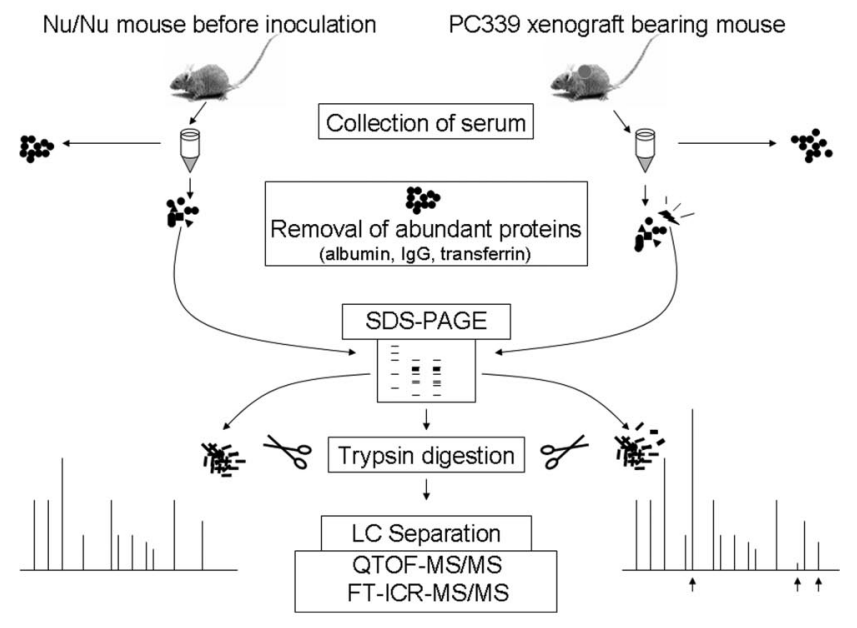

B

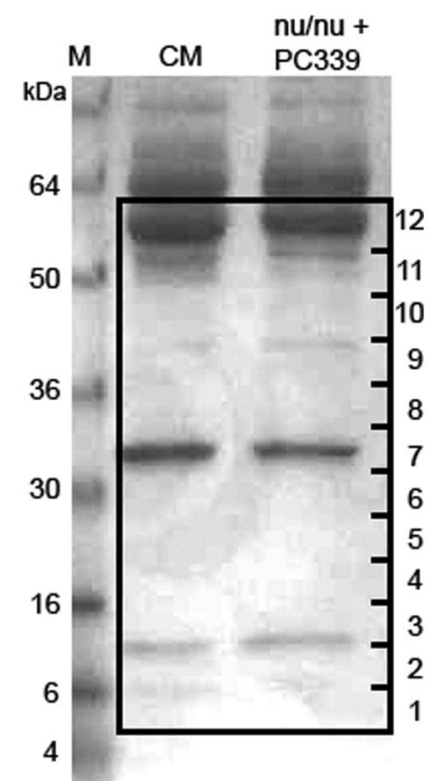

FIG. 1. Overview of the procedure. $A$, serum from control mice or mice carrying a PC339 prostate cancer xenograft was depleted of the most abundant proteins (albumin, transferrin, and immunoglobulins). Next the depleted fraction was separated on a gradient SDS-PAGE gel. The separated proteins were in-gel digested using trypsin. Then the peptides were separated by LC, and mass detection was performed by both Q-TOF- and FT-ICR-MS/MS. See "Experimental Procedures" for more details. $B$, Coomassie Brilliant Blue-stained gel used to produce the 12 different slices in each lane (indicated on the right). The human, tumor-derived proteins were found in gel slices 4 (NME2), 5 (NME1), 8 (TPI), 10 (GAPD, LDH-A, and LDH-B), 11 (ALDA), and 12 ( $\alpha$ enolase) of the nu/nu + PC339 serum sample. Mouse NME1 and mouse TPI were identified in gel slices 4 and 8, respectively, of the nu/nu control mouse (CM) serum sample. $M$ is marker lane. (See Table I and text for more details.)

As could be expected, most identified proteins are of mouse origin. However, using Q-TOF-MS/MS and FT-ICRMS/MS, we succeeded in identifying a limited number of 
TABLE I

Peptides identified by Q-TOF- and/or FT-ICR-MS/MS revealing the presence of human, tumor-derived proteins in serum of the xenograft-bearing mice

The letters in parentheses indicate that the peptide was found by either Q-TOF-MS/MS (Q), FT-ICR-MS/MS (F), or both (QF).

\begin{tabular}{|c|c|c|c|c|}
\hline & Mouse & Shared & Human & Coverage \\
\hline $\begin{array}{l}\text { Enolase, } \alpha \\
\text { Human P06733 } \\
\text { Mouse P17182 }\end{array}$ & YITPDQLADLYK (F) & IEEELGSK (F) & YISPDQLADLYK (F) & $20 / 433(5 \%)$ \\
\hline GAPD, liver & & VGVNGFGR (QF) & VIPELDGK (Q) & 95/334 (28\%) \\
\hline \multirow[t]{5}{*}{ Human P04406 } & & LTGMAFR (F) & VPTANVSVVDLTCR (QF) & \\
\hline & & & LISWYDNEFGYSNR (Q) & \\
\hline & & & VVDLMAHMASKE (F) & \\
\hline & & & GALQNIIPASTGAAK (F) & \\
\hline & & & IISNASCTTNCLAPLAK (F) & \\
\hline GAPD, muscle & & LTGMAFR (F) & VIPELDGK (Q) & 66/334 (20\%) \\
\hline \multirow[t]{4}{*}{ Human P00354 } & & & IISNASCTTNCLAPLAK (F) & \\
\hline & & & VVDLMAHMASKE (F) & \\
\hline & & & VPTANVSVLDLTCR (F) & \\
\hline & & & VGVDGFGR (QF) & \\
\hline TPI & SNVNDGVAQSTR (F) & IIYGGSVTGATCK (F) & SNVSDAVAQSTR (F) & $45 / 249(18 \%)$ \\
\hline Human P60174 & & TATPQQAQEVHEK (F) & & \\
\hline Mouse P17751 & & VVFEQTK (F) & & \\
\hline LDH-A & DQLIVNLLK (F) & $\operatorname{LNLVQR}^{a}(\mathrm{~F})$ & DQLIYNLLK (F) & $74 / 331(22 \%)$ \\
\hline Human P00338 & FIIPNIVK (F) & LVIITAGAR (F) & DYNVTANSK (F) & \\
\hline \multirow[t]{3}{*}{ Mouse P06151 } & VTLTPEEEAR (F) & VIGSGCNLDSAR $^{a}(\mathrm{~F})$ & FIIPNVVK $(F)$ & \\
\hline & & & QVVESAYEVIK (F) & \\
\hline & & & VTLTSEEEAR (F) & \\
\hline LDH-B & & SADTLWDIQK (Q) & LIAPVAEEEATVPNNK (F) & 93/333 (28\%) \\
\hline \multirow[t]{7}{*}{ Human P07195 } & & LNLVQR $^{a}(\mathrm{~F})$ & & \\
\hline & & IVVVTAGVR (F) & & \\
\hline & & VIGSGCNLDSAR $^{a}(\mathrm{~F})$ & & \\
\hline & & IVADKDYSVTANSK (F) & & \\
\hline & & FIIPQIVK (F) & & \\
\hline & & GLTSVINQK (F) & & \\
\hline & & IHPVSTMVK (F) & & \\
\hline ALDA & & VLAAVYK (F) & AAQEEYVK (F) & $104 / 364(29 \%)$ \\
\hline \multirow[t]{7}{*}{ Human P04075 } & & ALQASALK (F) & AAQEEYVKR (F) & \\
\hline & & QLLLTADDR (F) & PYQYPALTPEQK (F) & \\
\hline & & ALANSLACQGK (F) & & \\
\hline & & GILAADESTGSIAK (F) & & \\
\hline & & ADDGRPFPQVIK (F) & & \\
\hline & & ELSDIAHR (F) & & \\
\hline & & LQSIGTENTEENRR (F) & & \\
\hline PGAM1 & & HYGGLTGLNK (F) & & $32 / 253(13 \%)$ \\
\hline Human P18669 & & VLIAAHGNSLR (F) & & \\
\hline Mouse Q9DBJ1 & & HGESAWNLENR (F) & & \\
\hline NME1 & NIIHGSDSVK ${ }^{b}(F)$ & TFIAIKPDGVQR ${ }^{c}$ (QF) & DRPFFAGLVK (QF) & 87/152 (57\%) \\
\hline Human P15531 & FLQASEDLLK (F) & FEQKGFR $^{c}(\mathrm{Q})$ & NIIHGSDSVESAEK ${ }^{d}(F)$ & \\
\hline \multirow[t]{3}{*}{ Mouse P15532 } & & GLVGEIIKc (QF) & FMQASEDLLK (F) & \\
\hline & & VMLGETNPADSKPGTIR $(F)$ & & \\
\hline & & GDFCIQVGR $^{c}(\mathrm{~F})$ & & \\
\hline NME2 & NIIHGSDSVESAEK ${ }^{d}(\mathrm{~F})$ & TFIAIKPDGVQR ${ }^{c}$ (QF) & NIIHGSDSVK ${ }^{b}(\mathrm{~F})$ & 66/152 (43\%) \\
\hline Human P22392 & & FEQKGFR $^{c}(\mathrm{Q})$ & & \\
\hline \multirow[t]{4}{*}{ Mouse Q01768 } & & GLVGEIIKc (QF) & & \\
\hline & & VMLGETNPADSKPGTIR $^{c}(\mathrm{~F})$ & & \\
\hline & & GDFCIQVGR $^{c}(\mathrm{~F})$ & & \\
\hline & & DRPFFPGLVK (QF) & & \\
\hline
\end{tabular}

\footnotetext{
${ }^{a}$ Peptides present in both mouse and human LDH-A and LDH-B.

${ }^{b}$ Peptides present in both mouse NME1 and human NME2.

${ }^{c}$ Peptides present in both human and mouse NME1 and NME2.

${ }^{a}$ Peptides present in both mouse NME2 and human NME1.
} 
FIG. 2. Scheme of the glycolytic pathway showing the key enzymes and substrates. The enzymes ALDA, $\mathrm{LDH}-\mathrm{A}$ and $-\mathrm{B}$ were identified in the serum of the PC339-bearing mice. All except PGAM1 could be identified as human, tumor-derived proteins. TPI, GAPD, PGAM1, $\alpha$ enolase, and

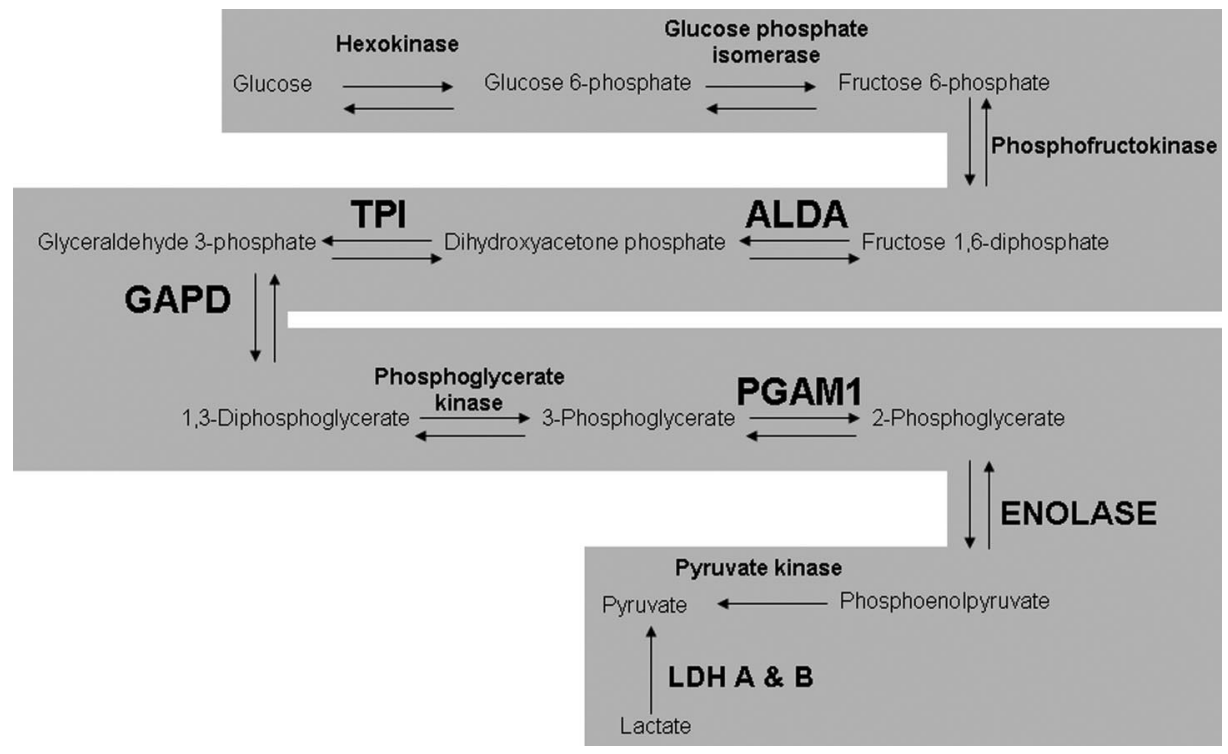

proteins as human, i.e. derived directly from the tumor. The mouse- and human-specific peptide sequences were blasted against $\mathrm{NCBI}$ databases, and none of them were observed in any other protein than the one annotated in the Swiss-Prot and IPI databases.

Identification of Human Glycolytic Proteins - In the PC339 serum, six different glycolytic enzymes were unambiguously identified as human: ALDA, TPI, GAPD, $\alpha$ enolase, LDH-A, and LDH-B (Table I and Fig. 2). The most upstream enzyme in the glycolytic pathway that was identified is ALDA. The 47$\mathrm{kDa}$ protein catalyzes the conversion of fructose 1,6-diphosphate to dihydroxyacetone phosphate. Positive identification of human ALDA was based on 11 peptides in fragment 11 of the gel (Fig. 1B) of which three were specific for the human protein. No mouse-specific peptides were observed in the xenograft sample (Table I). Two peptides of ALDA (GILAADESTGSIAK and LQSIGTENTEENRR) were also observed in the corresponding gel fragment of the control mouse.

In gel fragment 8 of the PC339 serum sample (Fig. 1B), human TPI was identified by FT-ICR-MS/MS. TPI ( 27 kDa) catalyzes the interconversion of glyceraldehyde 3-phosphate and dihydroxyacetone phosphate. Identification was based on four peptides, one of which was specific for the human protein. In addition, one mouse-specific TPI peptide was identified in the PC339 serum (Table I). The same peptide was also found in the corresponding gel fragment of the control serum sample.

In gel fragment 10 of the PC339 serum sample (Fig. 1B), both human liver and muscle GAPD were found. GAPD is a glycolytic enzyme with a molecular mass of $\sim 36 \mathrm{kDa}$ that catalyzes the conversion of D-glyceraldehyde 3-phosphate to 1,3-bisphosphoglycerate. Four peptides of liver GAPD were found by Q-TOF-MS/MS of which three were specific for the human protein. FT-ICR-MS/MS analysis revealed two of these as well as three additional peptides; the latter were all specific for human liver GAPD (Table I). Human muscle GAPD was identified by Q-TOF-MS/MS based on two unique peptides. FT-ICR-MS/MS analysis resulted in the identification of one identical and four additional peptides of which four were human-specific. No mouse-specific peptides were detected for either GAPD protein (Table I), nor were any of the GAPD peptides found in the control mouse serum.

Three peptides of the glycolytic enzyme phosphoglycerate mutase 1 (PGAM1) ( 29 kDa) were detected in gel fragment 8 of the PC339 serum sample (Fig. $1 B$ and Table I). Based on the three identified peptides, the distinction between human or mouse origin could not be made. PGAM1 is a highly conserved protein: only one of the 254 amino acids is distinct between mouse and human, making discrimination complicated. In the control serum, two PGAM1 peptides (HYGGLTGLNK and VLIAAHGNSLR) were found.

Furthermore two peptides of $\alpha$ enolase were discovered by FT-ICR-MS/MS in gel fragment 12 of the PC339 sample (Fig. $1 B$ and Table I). One of these peptides was human-specific. In addition, one peptide specific for mouse $\alpha$ enolase was detected in both the control and PC339 serum samples (Table I). $\alpha$ enolase (47 kDa) catalyzes the conversion of 2-phosphoglycerate to phosphoenolpyruvate.

Also both LDH-A and LDH-B were identified in gel fragment 10 of the PC339 serum sample (Fig. $1 B$ and Table I). LDH-A and $\mathrm{LDH}-\mathrm{B}$ are $37-\mathrm{kDa}$ enzymes that catalyze the conversion of lactate to pyruvate in the final step of the anaerobic glycolytic pathway. Five peptides specific for human LDH-A, three peptides specific for mouse LDH-A, and three peptides shared by both orthologues were found. For LDH-B, only one peptide specific for the human protein was observed, and eight peptides that are found in both the mouse and human protein were observed (Table I). The corresponding gel fragment of the control serum sample contained three mouse- 
A

FIG. 3. Identification of human NME1. $A$, FT-ICR-MS/MS spectrum of the FMQASEDLLK and DRPFFAGLVK peptides. The amino acid sequences matched residues $40-49$ and 57-66, respectively, of human NME1. $B$, primary sequence comparison between human NME1, human NME2, and mouse NME1 and NME2. The amino acid sequences of eight NME1 peptides (shaded boxes) of which two were human-specific (printed in bold) could be identified by Q-TOF- and FT-ICR-MS/MS. Amino acids discriminating NME1 from NME2 and mouse from human are underlined. The mouse-specific peptide leading to identification of mouse NME2 is printed in italics (see Table I and text for more details).

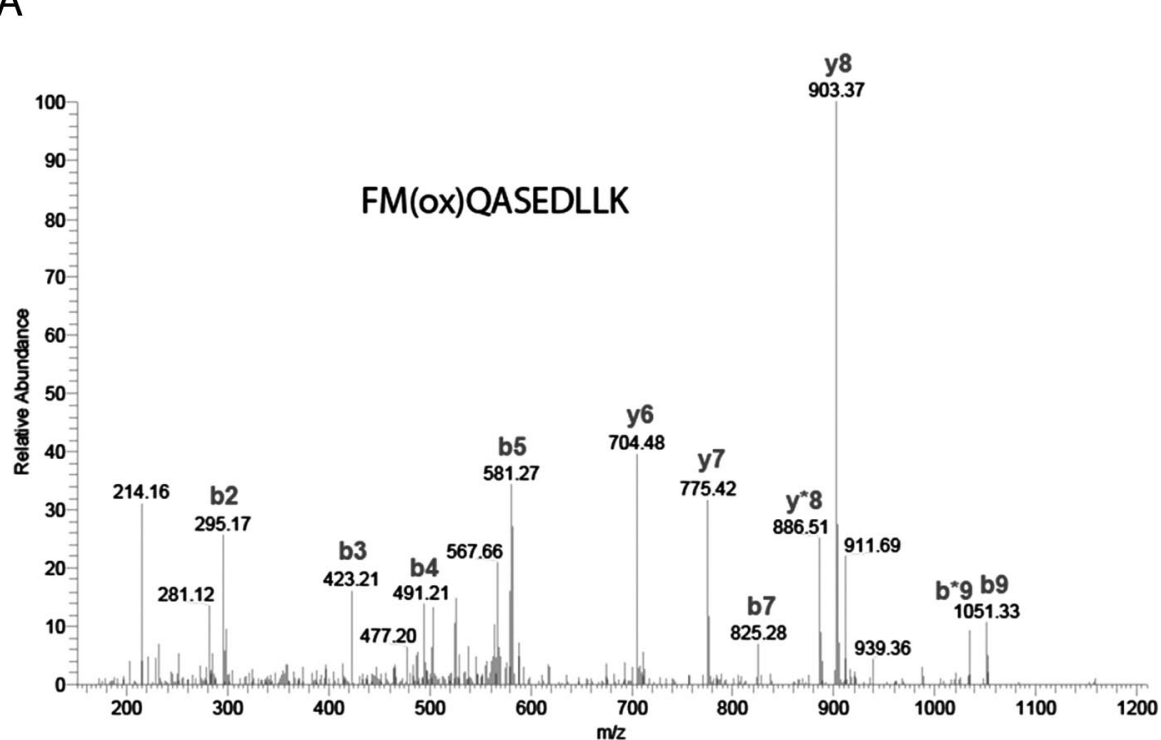

B

\begin{abstract}
HUMAN NME1 MANCERTFIAIKPDGVQR GLVGEIIKRFEQKGERLVGLKFMQASEDLLK
HUMAN NME2 MANLERTFIAIKPDGVQR GLVGEIIKREEQKGERLVAMKF LRASEEHLK

MOUSE NME1 MANSERTFIAIKPDGVQR GLVGEIIKREEQKGERLVGLKFLQASEDLLK

MOUSE NME2 MANLERTFIAIKPDGVQR GLVGEIIKREEQKGERLVAMKF LRASEEHLK
\end{abstract}

HUMAN NME1 EHYVDLKDRPFEAGLVKYMHSGPVVAMVWEGLNVVKTGRVMLGETNPAD

HUMAN NME2 QLHYIDLKDRPFFPGLVKYMNSGPVVAMVWEGLNVVKTGRVMLGETNPAD

MOUSE NME1 EHY TDLKDRPF FTGLVKYMHSGPVVAMVWEGLNVVKTGRVMLGETNPAD

MOUSE NME2 Q QHYIDLKDRPFFPGLVKYMNSGPVVAMVWEGLNVVKTGRVMLGETNPAD

HUMAN NME1 SKPGTIRGDFCIQVGR NIIHGSDSVESAEKEIGLWFHPEELVDYTSCAQNWIYE HUMAN NME2 SKPGTIRGDFCIQVGR NIIHGSDSVKKSAEKEISLLWFKPEE LVDYKSSCAHDWVYE MOUSE NME1 SKPGTIRGDFCIQVGR NIIHGSDSVKSAEKEISLWFEQPEE LVEYKSSCAQNWIYE MOUSE NME2 SKPGTIRGDFCIQVGR NIIHGSDSVESAEKEIHLWFKPEELIDYKSCAHDWVYE specific LDH-A peptides (FIIPNIVK, DQLIVNLLK, and VTLTPEEEAR), whereas no mouse-specific peptides for LDH-B were discovered.

Identification of Human NME-In gel fragment 5 of the PC339 serum sample (Fig. 1B), three peptides were identified by Q-TOF-MS/MS analysis (FEQKGFR, DRPFFAGLVK, and TFIAIKPDGVQR) that originate from NME. The peptides FEQKGFR and TFIAIKPDGVQR are present in both NME1 and NME2 from both mouse and man, making it impossible to determine whether these proteins originate from the host, the xenograft, or both. However, the DRPFFAGLVK peptide makes it possible to discriminate the protein as human NME1 because the mouse variant (Swiss-Prot accession number P15532) has a threonine, and both mouse and human NME2 contain a proline instead of alanine at the sixth position, respectively (Fig. 3). Proof of human origin of the NME1 protein was extended by analysis of the same sample by FT-ICRMS/MS, leading to identification of two of the same (DRPFFAGLVK and TFIAKPDGVQR) and five additional peptides (GLVGEIIK, GDFCIQVGR, NIIHGSDSVESAEK, VMLGETN- 
PADSKPGTIR, and FMQASEDLLK). The latter peptide is specific for human NME1. The NIIHGSDSVESAEK peptide is present in both human NME1 and mouse NME2 (Table I and Fig. 3).

Also in the PC339 mouse serum, in fragment 4 of the SDS-PAGE gel (Fig. 1B), three peptides were identified by Q-TOF-MS/MS analysis (DRPFFPGLVK, GLVGEIIK, and TFIAIKPDGVQR) that were found to be derived from NME2. The latter two peptides are also found in NME1, but DRPFFPGLVK is specific for NME2. However, the origin of the protein (mouse or human) could not be determined due to $100 \%$ interspecies homology of these peptides. To obtain more, potentially discriminating peptides, we performed FT-ICRMS/MS analysis on the same sample and found the same three but also five additional peptides (GDFCIQVGR, NIIHGSDSVK, NIIHGSDSVESAEK, VMLGETNPADSKPGTIR, and FLQASEDLLK). These peptides allow only positive identification of mouse NME1 (by the FLQASEDLLK peptide) and either one or both of human and mouse NME2 (Table I and Fig. 3). Although the NIIHGSDSVK peptide is only present in human NME2 and not in mouse NME2, we cannot rule out that this peptide originates from mouse NME1. Likewise the NIIHGSDSVESAEK peptide is only present in mouse NME2 and not in human NME2 but might also originate from human NME1 (Fig. 3).

Q-TOF-MS/MS analysis of the corresponding control mouse gel fragments revealed one NME peptide (TFIAIKPDGVQR). FT-ICR-MS/MS analysis produced four additional peptides (DRPFFPGLVK, GDFCIQVGR, GLVGEIIK, and NIIHGSDSVESAEK), confirming the presence of mouse NME2 in the serum of the control animals. No peptides specific for mouse NME1 were detected in the serum of the control animals.

Validation of Identified Proteins by Western Blot AnalysisThe presence of human GAPD in the PC339 serum sample was confirmed by Western blotting using a human-specific monoclonal antibody against GAPD. We could detect a signal at $\sim 38 \mathrm{kDa}$ in the serum of the PC339 xenograft mice but not in the control mice (Fig. 4A). To investigate GAPD expression variation among the pooled serum samples, the Western blotting experiments were repeated using the individual samples from both control and tumor-bearing groups. All nine xenograft serum samples expressed a strong GAPD signal, whereas no signal was observed in the nine control serum samples. In addition, a direct relationship was observed between tumor load and intensity of the GAPD signal (Fig. 4D). There was a moderate correlation between GAPD expression and tumor load ( $r=0.57$ ) (Fig. 4E).

We also verified our findings concerning the expression of NME by Western blotting. Clear protein bands of $\sim 18$ and 17 $\mathrm{kDa}$, corresponding to NME1 (Fig. 4B) and NME2 (Fig. 4C), respectively, were observed in the serum of the PC339 xenograft mice. In the serum of the control animals, no NME1 was detected, although a faint NME2 band, presumably due
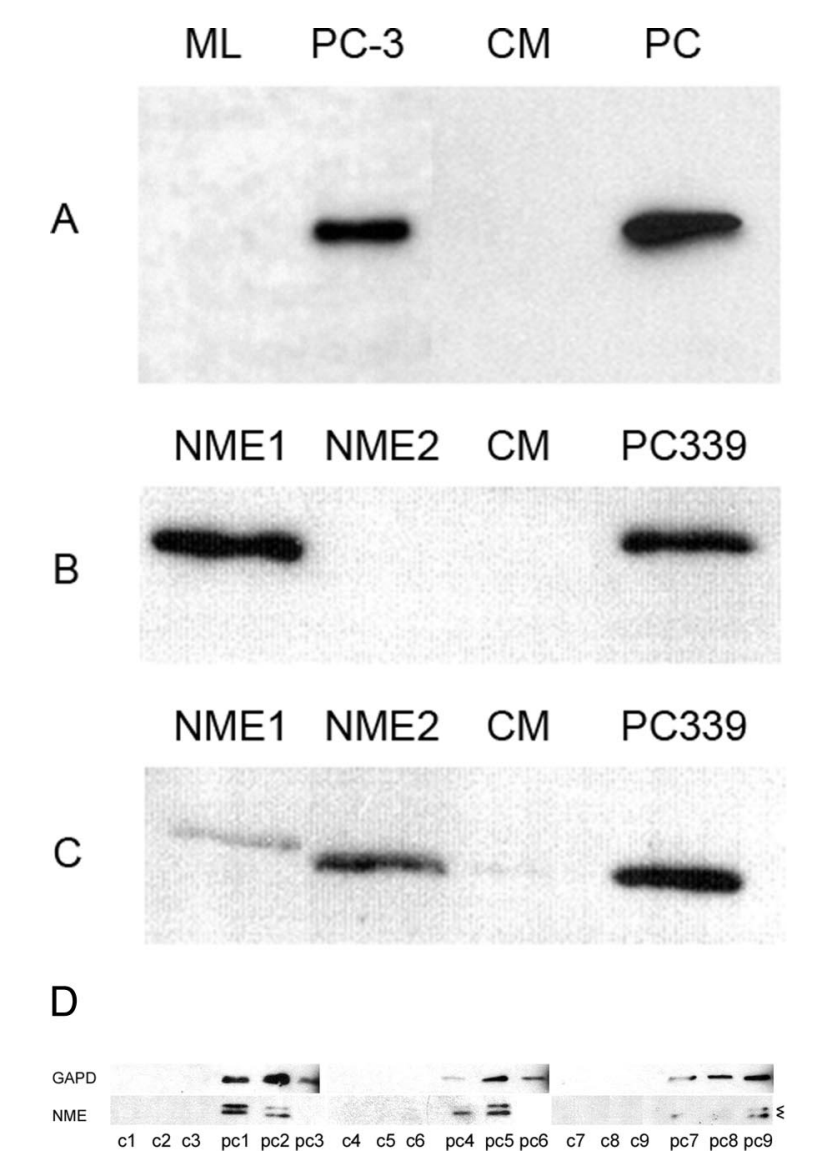

FIG. 4. Western blot detection of GAPD $(A), \operatorname{NME} 1(B)$, and NME2 $(C)$ in the pooled serum of nine control mice (CM) and nine PC339 xenograft-bearing mice $(P C)$. Normal mouse liver extract $(M L)$ and extract from human prostate cancer PC3 cells were used as negative and positive controls for human GAPD, respectively. NME1, recombinant human NME1; NME2, recombinant human NME2. $D$ shows Western blot detection of GAPD, NME1, and NME2 in nine individual control (c1-c9) and nine individual PC339 xenograft-bearing mice serum samples (pc1-pc9). Serum GAPD, NME1, and NME2 expression in the individual tumor-bearing mice was quantified by densitometry and was correlated to tumor weight $(E)$.

to cross-reactivity of the anti-human NME2 antibody with mouse NME2, was observed (Fig. 4C).

To investigate NME1 and NME2 expression variation among the pooled serum samples, the Western blotting experiments were repeated using the nine individual samples from both control and tumor-bearing groups. Six of nine xenograft serum samples expressed a strong signal for both NME1 and NME2 (Fig. 4D). There was a strong correlation between tumor load and intensity of the NME1 $(r=0.89)$ and NME2 $(r=0.73)$ signal (Fig. 4E). None of the control samples showed a signal for NME1. Three of nine control serum samples expressed a very faint NME2 signal.

\section{DISCUSSION}

In the present study we evaluated our novel proteomic approach to identify new potential biomarkers for prostate 
$E$
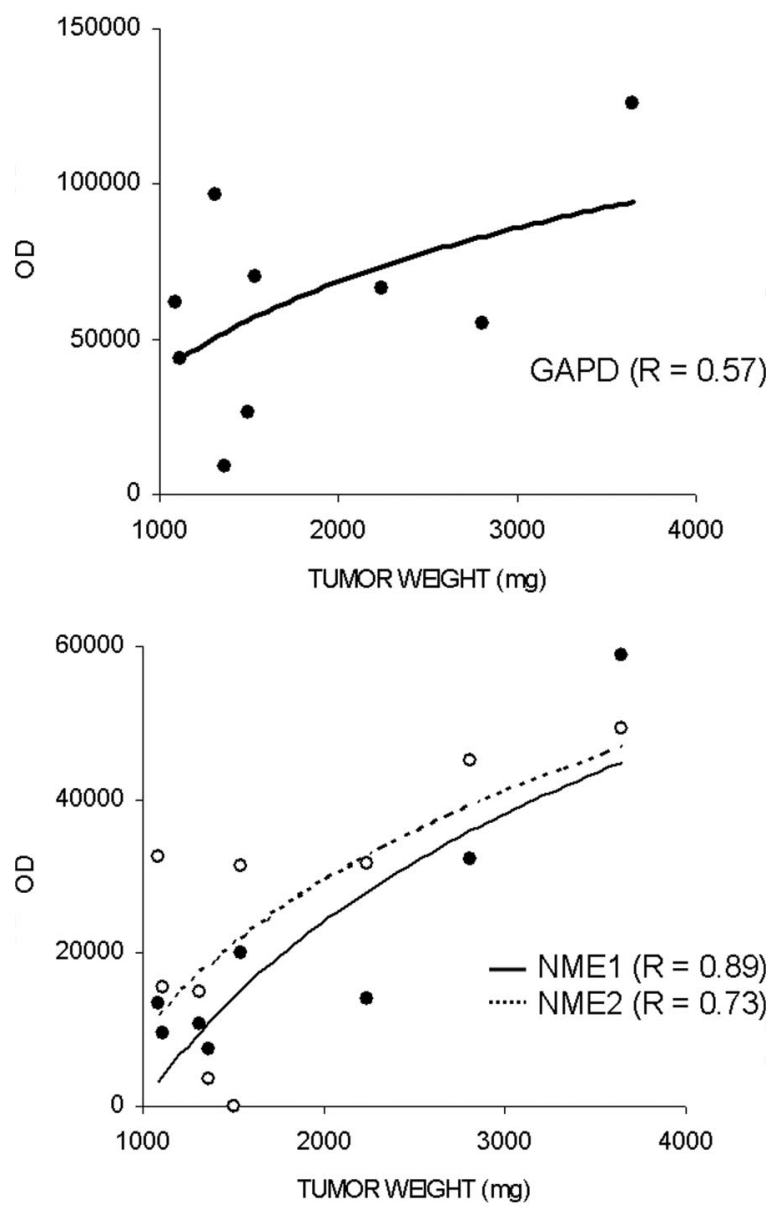

FIG. 4-continued

cancer diagnosis and progression. The use of a nude mouse as a host for human prostate cancer tissue in combination with sophisticated proteomic analytical techniques makes it feasible to specifically search for human proteins in the mouse serum. These are by definition derived from the tumor and thus might be of potential value as cancer biomarkers. As proof of concept, we report here the mass spectrometric identification of human NME and several human glycolytic enzymes in the serum of PC339 tumor-bearing nude mice.

In our approach we used two mass spectrometry techniques (Q-TOF and LTQ-FT-ICR). FT-ICR-MS/MS analysis revealed more unique proteins than did Q-TOF-MS/MS but more importantly also more distinct peptides per protein. Increasing the number of detected peptides per protein improves the confidence of protein identification. Because the sequence coverage of a certain protein clearly contributes to its positive identification and determination of its origin, the higher sensitivity and mass accuracy of FT-ICR-MS/MS proved to be a more effective technique than Q-TOF-MS/MS.

The large homology between human and mouse proteomes complicates the assignment of a protein as human (i.e. tumor- derived) or mouse. In database searches of control samples in human databases we could always identify a number of proteins (data not shown). Obviously these must be regarded as false positive identifications because by definition no human proteins were present in these samples. Also due to full homology of their identified peptides, we could not designate over 50 proteins as human or mouse by either Q-TOF- or FT-ICR-MS/MS (data not shown). The chance that performing the analysis twice using the identical sample will reveal additional discriminating peptides is small. We repeated the proteomic analysis of a gel part covering a molecular mass range of $\sim 35-60 \mathrm{kDa}$ and found no additional human proteins or peptides than the ones reported in the present study (i.e. GAPD, $\alpha$ enolase, ALDA, and LDH). Eighty-two percent of the identified proteins were found in both experiments (data not shown). However, other enrichment schemes might be fruitful in identifying additional human proteins (13). Still the amino acid sequence of a number of proteins is completely identical between the two species, and approaches to discriminate the two based on peptide mass will therefore not be effective. Obviously proteomic approaches like the one presented here strongly rely on high mass accuracy of the detected peptides but also on database accuracy and completeness (13).

The positive identification of human NME in the xenograftbearing mice clearly indicates that our approach is a promising tool to find new potential tumor markers. In humans, eight different genes of the NME family have been identified to date. The first NME gene, NME1 or nucleoside-diphosphate kinase A, was originally identified by differential hybridization of metastatic murine melanoma cell lines (14). Together with another NME family member, NME2, it forms an enzyme complex that transfers a phosphate among nucleoside tri- and diphosphates via an NME-phosphohistidine intermediate (15). Both human NME1 and NME2 genes are located on chromosome 17q21. Gain of the short arm of chromosome 17 is a frequently observed genetic abnormality in cancer that might contribute to the high expression of NME in a variety of human cancers. Also PC339 has gain of chromosome region $17 \mathrm{q} 21 .^{2}$

NME protein has been detected in the membrane, cytoplasmic, and nuclear fractions of cells. In addition, NME is secreted by tumor cells both in vitro (16) and in vivo (17-19). Compared with healthy control individuals, elevated expression of NME was measured in several body fluids, including bronchial lavage fluid of squamous cell lung cancer patients (17), and in serum and plasma of leukemia and non-Hodgkin lymphoma patients $(18,19)$.

In animal studies, NME expression is correlated with reduced metastasis of transplanted human cancer cells (2022). Also in a number of cancer patient cohort studies, the correlation between NME expression and metastatic potential of the tumor and/or patient prognosis and survival was addressed (for a review, see Ref. 10). A combination of reduced

\footnotetext{
${ }^{2}$ K. Hermans, unpublished observations.
} 
NME expression and high tumor metastatic potential was observed in several solid carcinomas. In contrast, other reports either show no or a positive correlation between NME expression and tumor metastasis and/or progression (Ref. 10 and references therein).

In prostate cancer, the significance of NME expression is still controversial. NME mRNA measurements and immunohistochemistry revealed strong expression levels in prostatic intraepithelial neoplasia and in prostatic adenocarcinomas, whereas expression in benign epithelium was much weaker $(23,24)$. NME1 may function as a suppressor of prostate cancer metastasis because its expression is lost in most metastatic lesions (25). In addition, a negative correlation between NME1 expression and tumor stage and grade was found (26), although others reported the opposite $(27,28)$.

In addition to human NME, we identified human GAPD, TPI, ALDA, $\alpha$ enolase, and LDH-A and LDH-B in the serum of the tumor-bearing mice. The expression of tumor-derived, human glycolytic pathway enzymes in the PC339 serum is presumably related to the increased energy requirements in the growing tumors (29). GAPD and TPI are key regulatory enzymes of glycolysis generally considered to be expressed in the cytoplasm, although the presence of high GAPD levels in serum of patients with liver cirrhosis has been reported (30). Interestingly GAPD mRNA expression was found to be associated with breast cancer cell proliferation, breast cancer aggressiveness, and prostate cancer stage $(31,32)$.

The mechanism by which NME and the glycolytic proteins have left the tumor and entered the blood stream is not clear. Generally these proteins are not considered as proteins that are secreted into the bloodstream. However, we did observe mouse homologs of these proteins in the control mouse serum (see "Results" for details). Furthermore the Plasma Proteome Project database of the Human Proteome Organization (HUPO) shows presence of these proteins in normal human blood plasma (www.bioinformatics.med.umich.edu/). Macroscopic examination of the tumor and surrounding tissue showed no necrosis.

Also noteworthy is the differential expression of mouse crystallins: 13 members of this family of small heat shock proteins were identified in the control samples, whereas none were found in the PC339 xenograft-bearing mice. This obviously would represent a direct or indirect host response to the tumor. Interesting in this respect is the decreased expression of crystallin $\alpha \mathrm{B}$ reported in testicular and breast tumors compared with normal tissue $(33,34)$ and the finding that low expression of crystallin $\alpha \mathrm{B}$ is associated with worsened survival of ovarian cancer patients (35).

The positive identification of human NME, a tumor-derived protein with potential diagnostic and/or prognostic value in prostate cancer, and several other human proteins that have been associated with cancer indicates that our concept of a proteomic approach in the prostate cancer xenograft nude mouse model is promising and will provide potential new biomarkers. The multitude and complexity of the proteins contained in body fluids still represent a major obstacle when searching for disease-specific markers. We used immunodepletion to remove the three most abundant proteins (albumin, immunoglobulins, and transferrin) from the mouse serum. On the one hand, this enables the detection of less abundant proteins, but on the other hand, this results in loss of potentially relevant proteins and peptides bound to these high abundance proteins (Ref. 36 and data not shown). To identify more xenograft-derived peptides/proteins, we will further optimize various steps in our protocol including depletion of other high abundance serum proteins, two-dimensional LC separation, and MS/MS with selected reaction monitoring. These enhancements will facilitate the identification of low abundance proteins in high and low molecular weight serum compartments. In general, the proteomic search for biomarkers in a xenograft model is not restricted to prostate cancer but can be applied to biomarker research in a wide variety of cancers and other diseases.

Acknowledgments - We are indebted to Dr. Wytske van Weerden for offering expertise in the xenograft mouse model and to Corrina de Ridder, Susan Reneman, Denie Zonruiter (Department of Urology), and Karel Bezstarosti (Department of Biochemistry) for technical assistance. Dr. Ioan Lascu (University of Bordeaux, Bordeaux, France) kindly provided the anti-human NME1 and NME2 antibodies and recombinant NME proteins. We thank the investigators at the analytical expertise center (Department of Biomolecular Mass Spectrometry, Utrecht University) of the Netherlands Proteomics Center for FT-ICR-MS analysis.

* This work was supported by the Netherlands Genomics Initiative (Horizon Breakthrough Project 050-71-106). The costs of publication of this article were defrayed in part by the payment of page charges. This article must therefore be hereby marked "advertisement" in accordance with 18 U.S.C. Section 1734 solely to indicate this fact.

${ }^{* \star}$ To whom correspondence should be addressed: Dept. of Urology, Rm. Be-362a, Josephine Nefkens Inst., Erasmus MC, P. O. Box 1738, 3000 DR Rotterdam, The Netherlands. Tel.: 31-104087672; Fax: 31-104089386; E-mail: g.jenster@erasmusmc.nl.

\section{REFERENCES}

1. Brawer, M. K. (1999) Prostate-specific antigen: current status. CA Cancer J. Clin. 49, 264-281

2. Schröder, F. H. (2005) Detection of prostate cancer: the impact of the European Randomized Study of Screening for Prostate Cancer (ERSPC). Can. J. Urol. 12, 2-6

3. Babaian, R. J., Johnston, D. A., Naccarato, W., Ayala, A., Bhadkamkar, V. A., and Fritsche, H. A., Jr. (2001) The incidence of prostate cancer in a screening population with a serum prostate specific antigen between 2.5 and $4.0 \mathrm{ng} / \mathrm{ml}$ : relation to biopsy strategy. J. Urol. 165, 757-760

4. Shariat, S. F., Canto, E. I., Kattan, M. W., and Slawin, K. M. (2004) Beyond prostate-specific antigen: new serologic biomarkers for improved diagnosis and management of prostate cancer. Rev. Urol. 6, 58-72

5. Van Weerden, W. M., de Ridder, C. M. A., Verdaasdonk, C. L., Romijn, J. C., Van der Kwast, T. H., Schröder, F. H., and Van Steenbrugge, G. J. (1996) Development of seven new human prostate tumor xenograft models and their histopathological characterization. Am. J. Pathol. 149, 1055-1062

6. Van Weerden, W. M., and Romijn, J. C. (2000) Use of nude mouse xenograft models in prostate cancer research. Prostate 43, 263-271

7. Roemer, I., Vogel, T., Otto, A., Fichtner, I., and Klose, J. (2001) Analysis of mouse beta-haptoglobin chain by lectin affinoblotting detection. Electrophoresis 22, 3038-3042 
8. Juan, H. F., Chen, J. H., Hsu, W. T., Huang, S. C., Chen, S. T., Yi-Chung Lin, J., Chang, Y. W., Chiang, C. Y., Wen, L. L., Chan, D. C., Liu, Y. C., and Chen, Y. J. (2004) Identification of tumor-associated plasma biomarkers using proteomic techniques: from mouse to human. Proteomics 4, 2766-2775

9. Hood, B. L., Lucas, D. A., Kim, G., Chan, K. C., Blonder, J., Issaq, H. J., Veenstra, T. D., Conrads, T. P., Pollet, I., and Karsan, A. (2005) Quantitative analysis of the low molecular weight serum proteome using ${ }^{18} \mathrm{O}$ stable isotope labeling in a lung tumor xenograft mouse model. J. Am. Soc. Mass Spectrom. 16, 1221-1230

10. Hartsough, M. T., and Steeg, P. S. (2000) Nm23/nucleoside diphosphate kinase in human cancers. J. Bioenerg. Biomembr. 32, 301-308

11. Ouatas, T., Salerno, M., Palmieri, D., and Steeg, P. S. (2003) Basic and translational advances in cancer metastasis: Nm23. J. Bioenerg. Biomembr. 35, 73-79

12. Krijgsveld, J., Ketting, R. F., Mahmoudi, T., Johansen, J., Artal-Sanz, M., Verrijzer, C. P., Plasterk, R. H., and Heck, A. J. (2003) Metabolic labeling of C. elegans and D. melanogaster for quantitative proteomics. Nat. Biotechnol. 21, 927-931

13. Nesvizhskii, A. I., and Aebersold, R. (2005) Interpretation of shotgun proteomics data: the protein inference problem. Mol. Cell. Proteomics 4, 1419-1440

14. Steeg, P. S., Bevilacqua, G., Kopper, L., Thorgeirsson, U. P., Talmadge, J. E., Liotta, L. A., and Sobel, M. E. (1988) Evidence for a novel gene associated with low tumor metastatic potential. J. Natl. Cancer Inst. 80, 200-204

15. Gilles, A. M., Presecan, E., Vonica, A., and Lascu, I. (1991) Nucleoside diphosphate kinase from human erythrocytes. Structural characterization of the two polypeptide chains responsible for heterogeneity of the hexameric enzyme. J. Biol. Chem. 266, 8784-8789

16. Okabe-Kado, J., Kasukabe, T., Honma, Y., Hayashi, M., Henzel, W. J., and Hozumi, M. (1992) Identity of a differentiation inhibiting factor for mouse myeloid leukemia cells with NM23/nucleoside diphosphate kinase. Biochem. Biophys. Res. Commun. 182, 987-994

17. Huwer, H., Kalweit, G., Engel, M., Welter, C., Dooley, S., and Gams, E. (1997) Expression of the candidate tumor suppressor gene nm23 in the bronchial system of patients with squamous cell lung cancer. Eur. J. Cardiothorac. Surg. 11, 206-209

18. Okabe-Kado, J. (2002) Serum nm23-H1 protein as a prognostic factor in hematological malignancies. Leuk. Lymphoma 43, 859-867

19. Niitsu, N., Okamoto, M., Honma, Y., Nakamine, H., Tamaru, J. I., Nakamura, S., Yoshino, T., Higashihara, M., Hirano, M., and Okabe-Kado, J. (2003) Serum levels of the nm23-H1 protein and their clinical implication in extranodal NK/T-cell lymphoma. Leukemia 17, 987-990

20. Leone, A., Flatow, U., VanHoutte, K., and Steeg, P. S. (1993) Transfection of human nm23-H1 into the human MDA-MB-435 breast carcinoma cell line: effects on tumor metastatic potential, colonization and enzymatic activity. Oncogene 8, 2325-2333

21. Ma, D., Luyten, G. P., Luider, T. M., Jager, M. J., and Niederkorn, J. Y. (1996) Association between NM23-H1 gene expression and metastasis of human uveal melanoma in an animal model. Investig. Ophthalmol. Vis. Sci. 37, 2293-2301

22. Suzuki, E., Ota, T., Tsukuda, K., Okita, A., Matsuoka, K., Murakami, M.,
Doihara, H., and Shimizu, N. (2004) Nm23-H1 reduces in vitro cell migration and the liver metastatic potential of colon cancer cells by regulating myosin light chain phosphorylation. Int. J. Cancer 108, 207-211

23. Myers, R. B., Srivastava, S., Oelschlager, D. K., Brown, D., and Grizzle, W. E. (1996) Expression of $\mathrm{nm} 23-\mathrm{H} 1$ in prostatic intraepithelial neoplasia and adenocarcinoma. Hum. Pathol. 27, 1021-1024

24. Jensen, S. L., Wood, D. P. Jr., Banks, E. R., Veron, M., Lascu, I., McRoberts, J. W., and Rangnekar, V. M. (1996) Increased levels of nm23 $\mathrm{H} 1$ /nucleoside diphosphate kinase A mRNA associated with adenocarcinoma of the prostate. World J. Urol. 14, S21-S25

25. Konishi, N., Nakaoka, S., Tsuzuki, T., Matsumoto, K., Kitahori, Y., Hiasa, Y., Urano, T., and Shiku, H. (1993) Expression of nm23-H1 and nm23-H2 proteins in prostate carcinoma. Jpn. J. Cancer Res. 84, 1050-1054

26. Stravodimos, K., Constantinides, C., Manousakas, T., Pavlaki, C., Pantazopoulos, D., Giannopoulos, A., and Dimopoulos, C. (2000) Immunohistochemical expression of transforming growth factor $\beta 1$ and $\mathrm{nm}-23 \mathrm{H} 1$ antioncogene in prostate cancer: divergent correlation with clinicopathological parameters. Anticancer Res. 20, 3823-3828

27. Igawa, M., Rukstalis, D. B., Tanabe, T., and Chodak, G. W. (1994) High levels of nm23 expression are related to cell proliferation in human prostate cancer. Cancer Res. 54, 1313-1318

28. Shiina, H., Igawa, M., Shigeno, K., Wada, Y., Yoneda, T., Yagi, H., Shirakawa, R., and Nagasaki, M. (1997) Relationship of serum testosterone level with proliferating cell nuclear antigen and nm23 protein in human prostatic carcinoma tissue. Oncology 54, 482-489

29. Tokunaga, K., Nakamura, Y., Sakata, K., Fujimori, K., Ohkubo, M., Sawada, K., and Sakiyama, S. (1987) Enhanced expression of a glyceraldehyde3 -phosphate dehydrogenase gene in human lung cancers. Cancer Res. 47, 5616-5619

30. Shibuya, A., and Ikewaki, N. (2002) High serum glyceraldehyde-3-phosphate dehydrogenase levels in patients with liver cirrhosis. Hepatol. Res. 22, 174-179

31. Révillion, F., Pawlowski, V., Hornez, L., and Peyrat, J. P. (2000) Glyceraldehyde-3-phosphate dehydrogenase gene expression in human breast cancer. Eur. J. Cancer 36, 1038-1042

32. Rondinelli, R. H., Epner, D. E., and Tricoli, J. V. (1997) Increased glyceraldehyde-3-phosphate dehydrogenase gene expression in late pathological stage human prostate cancer. Prostate Cancer Prostatic Dis. 1, 66-72

33. Klemenz, R., Scheier, B., Muller, A., Steiger, R., and Aoyama, A. (1994) Alpha $B$ crystallin expression in response to hormone, oncogenes and stress. Verh. Dtsch. Ges. Pathol. 78, 34-35

34. Takashi, M., Katsuno, S., Sakata, T., Ohshima, S., and Kato, K. (1998) Different concentrations of two small stress proteins, $\alpha \mathrm{B}$ crystallin and HSP27 in human urological tumor tissues. Urol. Res. 26, 395-399

35. Stronach, E. A., Sellar, G. C., Blenkiron, C., Rabiasz, G. J., Taylor, K. J., Miller, E. P., Massie, C. E., Al-Nafussi, A., Smyth, J. F., Porteous, D. J., and Gabra, H. (2003) Identification of clinically relevant genes on chromosome 11 in a functional model of ovarian cancer tumor suppression. Cancer Res. 63, 8648-8655

36. Zhou, M., Lucas, D. A., Chan, K. C., Issaq, H. J., Petricoin, E. F. , III, Liotta, L. A., Veenstra, T. D., and Conrads, T. P. (2004) An investigation into the human serum "interactome". Electrophoresis 25, 1289-1298 